\title{
Salivary Gland Disorder
}

National Cancer Institute

\section{Source}

National Cancer Institute. Salivary Gland Disorder. NCI Thesaurus. Code C26879.

A non-neoplastic or neoplastic (benign or malignant) disorder involving a salivary gland. 